\title{
Rapid Detecting Total Acid Content and Classifying Different Types of Vinegar based on Near Infrared Spectroscopy and Ant Colony Optimization Partial Least-Squares Analysis
}

\author{
Zhu Yao-Di ${ }^{\mathrm{a}}$, Zou Xiao-Bo, ${ }^{\mathrm{a}, \mathrm{b}^{*}}$, Huang Xiao-Wei ${ }^{\mathrm{a}}$, Shi Ji-Yong ${ }^{\mathrm{a}}$, Zhao Jie-Wen ${ }^{\mathrm{a}}$, Li Yanxiao ${ }^{\mathrm{a}}$, \\ Hao Limin ${ }^{c}$ and Zhang Jianchun ${ }^{c}$
}
${ }^{a}$ School of Food and Biological Engineering, Jiangsu university, 301 Xuefu Rd., 212013 Zhenjiang, Jiangsu, China
${ }^{b}$ Key Laboratory of Modern Agricultural Equipment and Technology, 301 Xuefu Rd., 212013 Zhenjiang, Jiangsu, China

${ }^{c}$ The Research Center of China Hemp Materials, Beijing, China

\begin{abstract}
More than 3.2 million litres of vinegar is consumed every day in China. Traditional Chinese vinegars are prepared through solid-state fermentation (SFF) and made from different sorts of cereals. Chinese vinegars have specific local features. Every region has its own manufacturers, who produce vinegar in specific processes, using particular raw materials. How to control the quality of vinegar is problem. Near infrared spectroscopy (NIR) transmission technique was applied to achieve this purpose. 46 traditional vinegar samples were collected. They were classified into Sanxi vinegar, Zhenjiang vinegar, Micu vinegar, and Baonin vinegar according to their origin. Micu vinegar and Baonin vinegar were separated from the other categories in the two-dimension principal component space of NIR after principle component analysis (PCA). Ant colony optimization partial least-squares analysis (ACO-PLS) was firstly applied to identify the four categories vinegar. The accuracies of identification were more than $85 \%$. As total acid content (TAC) is highly connecting with the quality of vinegar, NIR was used to predicate the TAC of samples. ACO-PLS was applied to building the TAC prediction model based on spectral transmission rate. Compared with full spectral partial least-square (PLS) model, ACO-PLS model gave better precision and accuracy in predicting TAC. The determination coefficient for prediction $\left(R_{p}\right)$ of the ACO-PLS model was 0.921 and root mean square error for prediction (RMSEP) was 0.3031 . This work demonstrated that near infrared spectroscopy technique coupled with ACO-PLS could be used as a quality control method for vinegar.
\end{abstract}

Keywords: Near infrared spectroscopy, Ant colony optimization, Vinegar, Total acid content, Principle component analysis, Partial least-square.

\section{INTRODUCTION}

Vinegar is an important element in Asian, European, Western, and other traditional cuisines of the world [1]. The Codex Alimentarius (1987) Commission of $\mathrm{FAO} / \mathrm{WHO}$ joint commission defined vinegar as a liquid fit for human consumption, produced from a suitable raw material of agricultural origin, containing starch, sugars or starch and sugars, by the process of double fermentation, alcoholic and acetous, that can constrains a specified amount of acetic acid [2]. Many medicinal components that are good for health have been reported in vinegar, such as carbohydrates [3], organic acids (acetic, formic, lactic etc) [4], alcohols and polyols (ethanol, acetoin, 2,3-butanediol and hydroxymethylfurfural), amino acids and peptides, and volatile substances (ethyl acetate). Many remedies and treatments have been ascribed to vinegar, and great effort has been focused on identifying the functional

\footnotetext{
*Address correspondence to this author at the Key Laboratory of Modern Agricultural Equipment and Technology, 301 Xuefu Rd., 212013 Zhenjiang, Jiangsu, China; Tel: +86 511 88780174; Fax: +86 511 88780201;

E-mail: zou_xiaobo@ujs.edu.cn
}

components in vinegar and their reaction mechanisms [5].

China has more than 5000 year's history of producing vinegar. Every year over 26 million hectolitres of vinegar is produced in China. Chinese people treat the vinegar as favourite condiments, health products and even medicines. More than 3.2 million litres of vinegar is consumed every day in China. There are many types of vinegar in China. How to control the quality of vinegar is problem.

Different types of vinegar have the same main components, water and acid. Vinegar contains many nutrients, which contains more acids, such as acetic acid, lactic acid, pyruvic acid, formic acid, malic acid, citric acid, oxaloacetate, and succinate et al. These amount nutrients are connecting with the total acidity content (TAC). That is, TAC is highly connecting with the quality of vinegar. The United States Food and Drug Administration (FDA) require that any product called "vinegar" contain at least $4 \%$ acidity. The Codex standard proposed a minimum of $6 \%$ for wine vinegar and $5 \%$ for others because the percent of acetic acid 
present in the product varies according to what they are made from [3].

Nowadays, the quantitative analysis of vinegar acidity is performed according to various wet chemical method such as high performance liquid chromatography (HPLC) [6, 7], gas chromatographic (GC) [4], capillary electrophoresis [8, 9] and chemical titration, etc. However, all of the methods mentioned above are time-consuming in the quantitative analysis of vinegar total acid. Near infrared spectroscopy is a fast, accurate, easy and non-destructive technique that can be as a replacement of time-consuming chemical method.

Recently, NIR spectroscopy has been applied for the discrimination of aging of vinegar during storage [10], and prediction of chemical constituents such as organic acids during storage and aging [11], reducing sugars [12], total procyanidins [13], soluble solids content and $\mathrm{pH}$ of rice vinegar [14] and discrimination of fruit vinegar varieties [15]. These researchers demonstrate that NIR could be a good method for quantity and quality control of vinegar. However, From the complete spectral data, which can be recorded for a given sample, it is likely that some of the signals may not be selective as regards the property of interest, while some others may be only partially selective. Hence, variables are usually subjected to a careful selection process before submitting them to PLS regression. This means that the multivariate model is built with only a limited number of signals. The purpose of variable selection is the obtainment of models based on spectral data carrying a higher information content as regards the analyte or property of interest [16]. Additionally, less spectral overlapping with interferences is sought. Improved PLS analytical performance has been reported upon variable selection, which supports the continuing interest in this chemometric activity [17]. The subject has been recently reviewed, with particular emphasis on NIR spectroscopic applications.

Ant colony optimization (ACO) has been introduced for variable selection in PLS regression problems (ACO-PLS) [2, 18-20]. ACO resembles the behavior of ant colonies in the search for the best path to food sources. Variables are identified with space dimensions defining the available paths followed by ants, with allowed coordinates of 1 or 0 (selected and unselected features respectively). In this way, a given path is connected to a number of selected variables, which in turns corresponds to a given prediction error. In each generation, ants deposit a certain amount of pheromone, which increases with decreasing values of the objective function defined by each path. They find new paths based on the following information: (1) the pheromone amount accumulated in each of the dimension coordinates, (2) a heuristic measure of path goodness, and (3) a random search across all available paths. Ant search is then based on a probabilistic combination of these factors, which allow deviations from the best looking paths [21].

The aim of this paper is to investigate the potential of ACO-PLS and near infrared spectroscopy technique for non-destructive measurement of Chinese vinegar. The ACO-PLS model will be used for predication TAC and classification of Chinese vinegar based on NIR spectra.

\section{MATERIALS AND METHODS}

\subsection{Sample Preparation}

Forty six traditional vinegar samples were collected from the industry and several supermarkets in China. All of these vinegars were commonly used in Chinese people's daily life. Before the experiment, the vinegar samples were stored in the laboratory at a constant temperature of $25 \pm 1^{\circ} \mathrm{C}$ for more than $48 \mathrm{~h}$ to have an equalization room temperature. The samples were all original vinegar liquid without dilution.

\subsection{Total Acid Content Measurement}

Reference analyses were in accordance with the Official Methods of Analysis for Chinese vinegar (GB/T 5009.41-2003) (GB means national standard; 5009.41 was the codes of the official methods; and 2003 was the year in which the methods were revised). Absorbed $5.0 \mathrm{ml}$ sample and placed in $100 \mathrm{ml}$ volumetric flask, add distilled water to the mark, mix and absorb $20.0 \mathrm{ml}$ then placed in $200 \mathrm{ml}$ beaker, add distilled water to $60 \mathrm{ml}$, starting magnetic stirrer, titration with standard sodium hydroxide solution $[\mathrm{c}(\mathrm{NaOH})=0.05 \mathrm{~mol} / \mathrm{L}]$ titrated to $\mathrm{pH}=8.2$ which shows in $\mathrm{pH}$ meter and recorded consumption of standard sodium hydroxide solution $(0.05 \mathrm{~mol} / \mathrm{L})$ of $\mathrm{ml}$, then total acid content can be calculated. 3 groups of each sample measured in parallel, 3 groups of determinations of the maximum relative error is less than $3 \%$.

\subsection{Spectra Collection and Preprocessing}

The NIR spectra were collected in the transmission mode using an Antaris II type Fourier transform near 
infrared spectroscopy (Thermo Fisher, USA) with a standard glass colorimetric ware in the range from 10,000 to $4000 \mathrm{~cm}^{-1}$. The spectra used for the data analysis were the average spectra of sixteen times scans, and the data were measured in $1.928 \mathrm{~cm}^{-1}$ intervals, which results in 3112 variables. The temperature and humidity were kept a steady level in lab (temperature was $25 \pm^{\circ} \mathrm{C}$ and humidity was $60 \%$ ).

Raw vinegars near infrared transmission spectra shown in Figure 1, in which can be seen in the range of $4000-5300 \mathrm{~cm}^{-1}$ and $6500-7098 \mathrm{~cm}^{-1}$ spectral response value is almost 0 . Therefore, these two ranges spectra will be removed in the following processing to improve the modeling efficiency. Selected spectral as the final model range are $5301-6498 \mathrm{~cm}^{-1}$ and $7100-10000 \mathrm{~cm}^{-1}$, results in 2126 variables. Multiplicative scatter correction (MSC) was used to correct for additive and multiplicative effects in the vinegar spectra.

\subsection{Ant Colony Optimization}

The ACO creates a large population of artificial ants that search for the high dimensional spectral variable space in parallel for the best features suited for a particular discrimination problem. In this study, each artificial ant is assigned to a unique subset of variables in the NIR spectrum, and the artificial ants communicate by a virtual chemical pheromone trail distributed over the NIR variables that are changed dynamically at each run-time and reinforcing itself via positive feedback. To exclude those inefficient variables, an evaporation constant is imposed such that the function uniformly decreases the pheromone trail in real time. The ACO algorithm iteratively performs a loop containing the three central elements as follows: (i) Generation of ants that are stochastically assigned to spectral variables is based on the global pheromone trail; (ii) Each ant's performance is evaluated (i.e., classification accuracy of each subset of Raman shifts) and (iii) The global pheromone trail is updated via an adoption of the evaporation constant and the classification performance of the ants. These three steps are repeated reiteratively in search for the global optimum of the classification accuracy. Supposing there are $\mathrm{m}$ variables for selection and $k$ ants participating into the searching process, a brief description of ACO can be shown as below:

(1) Pheromone amount initialization: give each variable the same initial pheromone amount. $\tau_{i}(0)=\varnothing(i=1,2, \ldots \ldots, m)$.

(2) Solution selection: Each ant selects features based on a probability function. A simple but effective estimate of the probability of each variable to be selected can be:

$$
P_{i}(t)=\frac{\tau_{i}(t)}{\sum_{i=1}^{m} \tau_{i}(t)}
$$

Where $\tau_{i}(t)$ is the amount of pheromone at time $t$ for the ith variable. This selection process can be briefly described as: (1) accumulate the probabilities of all the features in such a way that $\operatorname{accu}(I)=\operatorname{accu}(i-1)+P(i)$, where $\operatorname{accu}(0)=0$. Clearly, $\operatorname{accu}(m)=1 \quad(m$ is the total feature number); (2) generate a random number between 0 and 1 , if the this random number is between $\operatorname{accu}(i-1)$ and $\operatorname{accu}(i)$, the th feature is selected. This process is also widely used in ant colony optimization algorithm formember(chromosome) selection. Clearly,

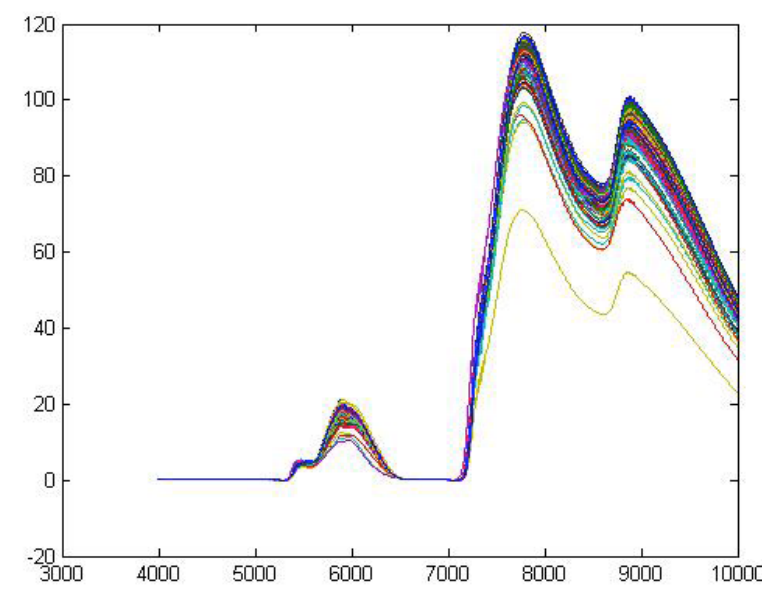

(a)

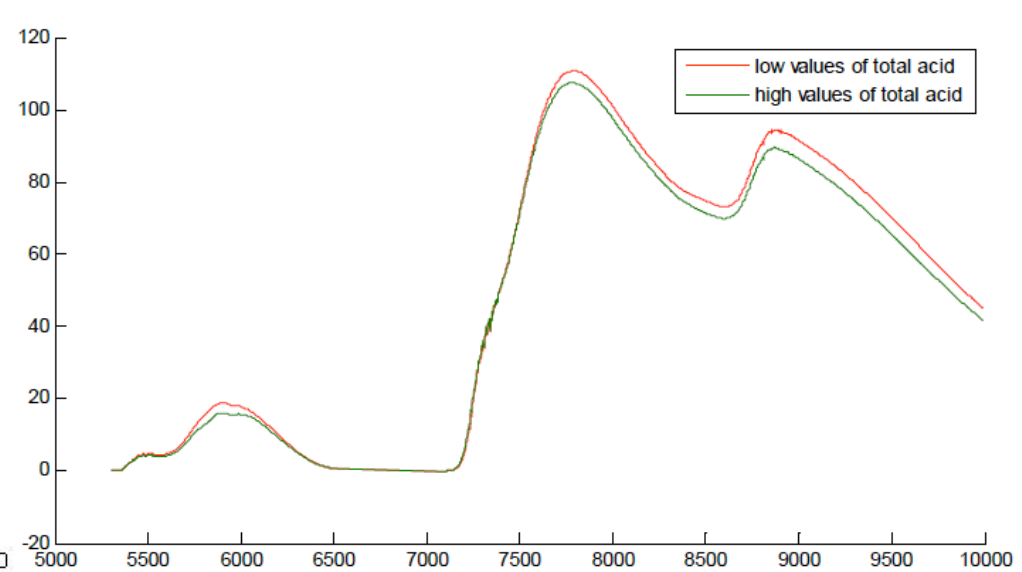

(b)

Figure 1: Chinese vinegar NIR spectra, (a) original NIR spectra of 46 samples, (b) mean spectrum of ten samples with highest $(6.621 \%)$ or lowest $(2.461 \%)$ total acid content. 
the variable with more pheromone is more likely to be selected. One can adjust the selection probability of each variable by employing weighting functions.

(3) Solution score evaluation: Set up a criterion or objective function for solution evaluation. In this study, classification accuracy based on a PLS classifier is used as a criterion to evaluate the quality of the selected variables of each ant.

(4) Pheromone amount updating: Pheromone amount of each variable is updated based on the equation below

$T_{i}(t+1)=r \cdot T_{i}(t)+\Delta T_{i}(t)$

where $i$ is variable index, $r$ is a constant between 0 and 1 , called pheromone remaining rate which is complementary to evaporation rate $e(r=1-e)$, and $\Delta T_{i}(t)$ represent pheromone increase, which is proportional to the classification accuracy of the selected features of each ant. For each ant, only the pheromone of the selected variables will increase. The higher the accuracy is, the more the pheromone increases. During the whole searching process, the pheromone of all the variables keeps evaporating based on a predefined evaporation rate $e$.

Repeat the above "selecting", "scoring", and "updating" steps for $k$ ants, which is called one iteration. Ideally, after a number of iterations, all the ants will be convergent to the same set of features, which theoretically is the optimal solution of the optimization problem.

\subsection{Software}

All algorithms were implemented with Matlab V7.4 (Mathworks, USA) under Windows XP. PLS algorithm (iToolbox, Lars Norgaard, Denmark) were used as a comparable methods for regression and classification. For the spectral acquisition Antaris II type Fourier transform near infrared spectroscopy (Thermo Fisher, USA) was used.

\section{RESULTS AND DISCUSSION}

\subsection{Vinegar Sample Investigation}

Traditional Chinese vinegars are prepared through solid-state fermentation (SFF) and made from different sorts of cereals, where as in more recent years many vinegars prepared from fruits have appeared on the market. The SSF process for Chinese vinegars comprises four successive stages, namely koji preparation, saccharification of starch and alcohol fermentation, acetic-acid fermentation, and maturation. Chinese vinegars have specific local features. Every region has its own manufacturers, who produce vinegar in specific processes, using particular raw materials. Consequently, each vinegar has its own taste, flavor characteristic, and market. More information about Chinese could be seen in reference articles $[1,12,14$, 22]. According to their origin, the 46 samples were divided into Sanxi vinegar, Zhenjiang vinegar, Micu vinegar, and Baonin vinegar.

\subsection{Spectra Investigation}

The raw absorbance spectra of vinegars are shown in Figure 1. There were two main features of the absorbance spectra around wavenumbers $8873 \mathrm{~cm}^{-1}$ and $7797 \mathrm{~cm}^{-1}$, which related to the second overtone of the $\mathrm{C}-\mathrm{H}$ stretch and a combination of $\mathrm{O}-\mathrm{H}$ stretching and $\mathrm{C}-\mathrm{H}$ stretching, respectively [23]. Peak at $5910 \mathrm{~cm}^{-1}$ might be absorption of the $\mathrm{C}-\mathrm{H}$ stretch first overtone. Small absorbance peak at $5511 \mathrm{~cm}^{-1}$ might be associated with glucose, sucrose, fructose and amino acid in vinegars [15]. The reference values of total acid were different and had a broad range of variation from each variety of vinegars, which indicated that the internal qualities of these vinegars were different.

In Figure 1a, the vinegars spectra were analogue thanks to the hydrogen bond in these samples. It could be seen that the main component in vinegar samples is water $(>90 \%, \mathrm{~g} / 100 \mathrm{ml})$. However, the hydrogen-based group of acids in vinegars could also influence the spectra. Figure 1b shows two mean spectrum, which represent the "Zhenjiang Xiangcu" with the highest $(6.443 \%, \mathrm{~g} / 10 \mathrm{ml})$ and "MiCu" the lowest $(2.595 \%$, $\mathrm{g} / 100 \mathrm{ml}$ ) total acid content. It could be conclude that the higher acid content the more absorbing in spectra.

\subsection{Principal Components Analysis (PCA)}

All NIR spectral data from 46 vinegar samples were used for the PCA. Although PCA itself cannot be used as a classification tool, this behavior may indicate the data trend in visualizing dimension spaces. The scatter plots of data using the top two principal components (PCs) issued from PCA were obtained as showed in Figure 2. The accumulated variance contribution rate was up to $96.12 \%$ for the top two PCs, in other words, the space represented by PCs 1-2 vectors can explain $96.12 \%$ chemical information in the NIR spectroscopy 
region. Thus, the $2 \mathrm{D}$ space can almost express fully the information that all data are distributed in an ultradimensional space. The Micu vinegar and Baonin vinegar were separated from the other two vinegars in the two-dimension principal component space. The Sanxi vinegar, Zhenjiang vinegar, Micu vinegar, and Baonin vinegar were all prepared thorough the solidstate fermentation processing. However, the raw materials and processing of different vinegars were different. Zhenjiang aromatic vinegar is most famous in southern China. Its main raw materials are sticky rice, but some sugar are added in the maturate process. Shanxi vinegar is the most famous vinegar in northern China, and use sorghum as the main raw material. Some herbs are used as the raw materials for Sichuan vinegar production. For Micu vinegar, mainly collected from Zhejiang and Fujian province in China, pigmented microorganisms (mainly Monascus) predominate in the fermentation process and give the rose colour of the vinegar. Further investigation in the total acid content (TAC) of each sample in Figure 2 , it could be seen that the TAC increase with the value of PC2 in the same category. Therefore, in the NIR spectroscopy region, these spectral differences provided enough information for further quantitative and qualitative analysis.

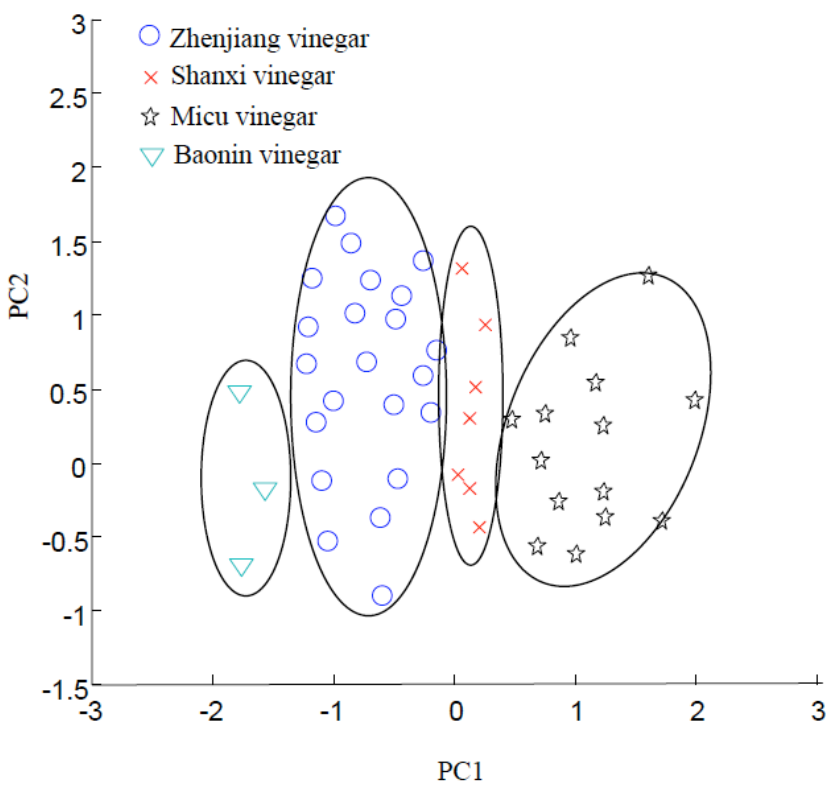

Figure 2: The scatter plots of data using the top two principal components (PCs) of PCA.

\subsection{ACO-PLS Classification Model}

As Micu vinegar and Baonin vinegar could be clearly classified from the rest categories, this study would classify Sanxi vinegar, Zhenjiang vinegar through ACO-PLS. In this experiment, there were 7
Shanxi vinegars, 21 zhenjiang vinegars. The ACO-PLS algorithms were developed for spectral feature selection to identify the useful NIR regions of vinegar spectra for classification vinegars. The algorithm parameters balanced the exploitation- exploration trade-off and were adjusted by trial and error (100 ants, $\alpha=1, \beta=1$ and $\rho=0.2$ ) to a high convergence rate for obtaining the best subset of NIR variables for vinegar distinction. Figure 3 shows the relationship of the error rate with respect to the evolution of the best ant of the respective ants generated, indicating a significant improvement in the classification accuracy of vinegars as the ACO algorithm progresses. Consequently, the termination criterion was chosen at 30 iterations as the best ant in most runs had converged to a high classification accuracy.

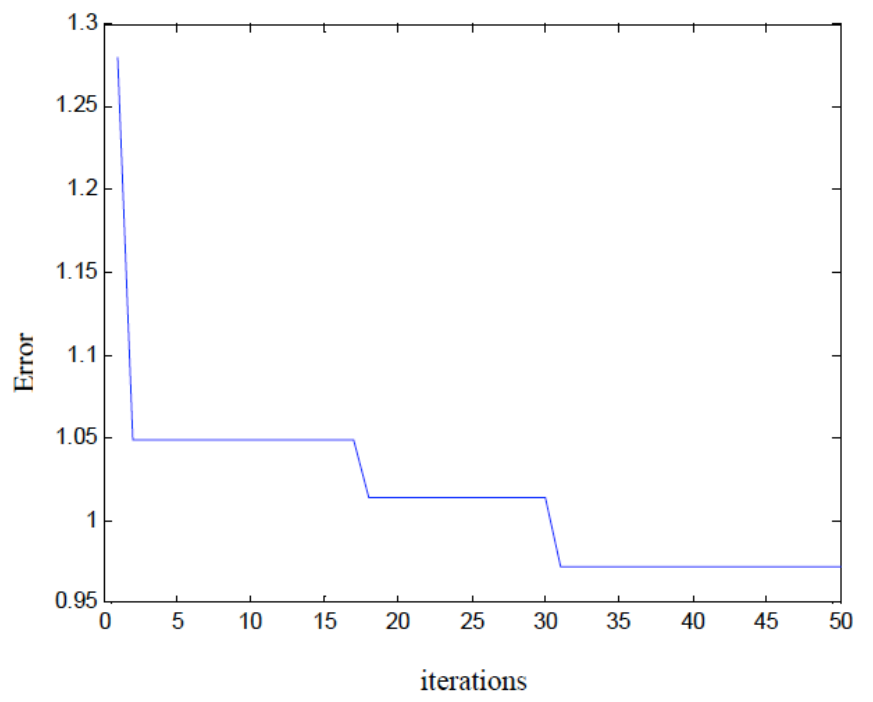

Figure 3: The mean classification error rates of the best performing ants for 50 iterations in ACO-PLS algorithms Parameters (100 ants, $\alpha=1, \beta=1$ and $\rho=0.2$ ).

Figure 4 shows the classification results of the ACO-PLS model based on the two prominent NIR spectra bands identified $\left(5714 \sim 6125 \mathrm{~cm}^{-1}, 8366\right.$ $8568 \mathrm{~cm}^{-1}$ ) together with the leave-one-out, cross validation. The ACO-PLS algorithm yielded an overall accuracy of $95.6 \%$ for classification. Hence, ACO-PLS provides a novel way to determine significant NIR spectral regions which are important towards constructing a final model for classification. We also evaluated the performance of the ACO-PLS algorithms using a new dataset (18 Zhenjiang vinegar and 12 Shanxi vinegar) as an independent prediction dataset. The predictive accuracy $96.7 \%$ could be achieved for the independent validation dataset, substantiating the robustness of the ACO-PLS algorithms developed for NIR spectra classification of vinegars. 


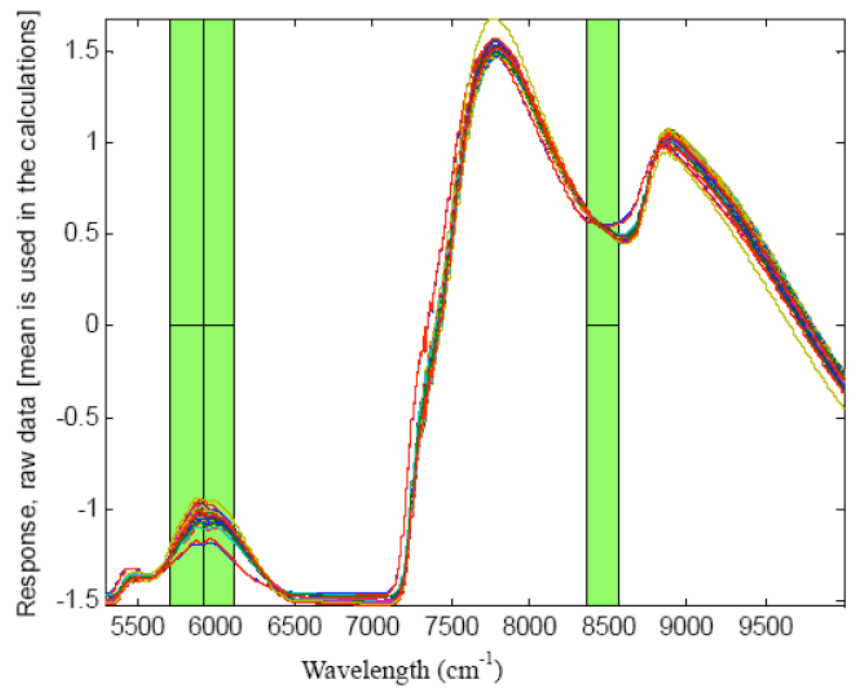

Figure 4: The most significant NIR spectra regions identified by the ACO-PLS algorithms for classification.

\subsection{ACO-PLS Regression Models}

The 46 NIR spectra were also used to build the predication models of total acid content (TAC) in vinegar. All 46 vinegar samples were divided into two subsets. One of it called calibration set was used to build model, and the other called prediction set was used to test the robustness of model. The mean value for TAC of samples in calibration set was $0.04478 \mathrm{~g}$. $\mathrm{ml}^{-1}$. These variables could be directly used to establish the regression model by PLS, Figure $\mathbf{5}$ shows the RMSECV values of PLS model under different PCs by cross-validation. When using $9 \mathrm{PCs}$, the lowest RMSECV could be achieved, and the corresponding PLS model was the optimal model. The PLS model performed on a new dataset (18 Zhenjiang vinegar and 12 Shanxi vinegar). The RMSEP and the $R$ of PLS model were 0.402 and 0.8754 in prediction set respectively. However, Variable selection in multivariate analysis is a very important step, because the removal of non-informative variables will produce better prediction and simpler models. It has been shown that the predictive ability can be increased and, the complexity of the model can be reduced by a judicious pre-selection of wavelengths.

The number of input variables is 2116 from the ranges of $5301-6498 \mathrm{~cm}-1$ and $7100-10000 \mathrm{~cm}-1$ for each sample. Thus, each ant in each ACO had 2116 elements. As a global selection method, it is very difficult to selection from such big elements. Therefore, the NIR spectra variables were divided into 20 regions for selection. As it is discussed in the theory section, to set optimized values to $\alpha, \beta$ and $\rho$ parameters, a 3levels full factorial experimental design involving these

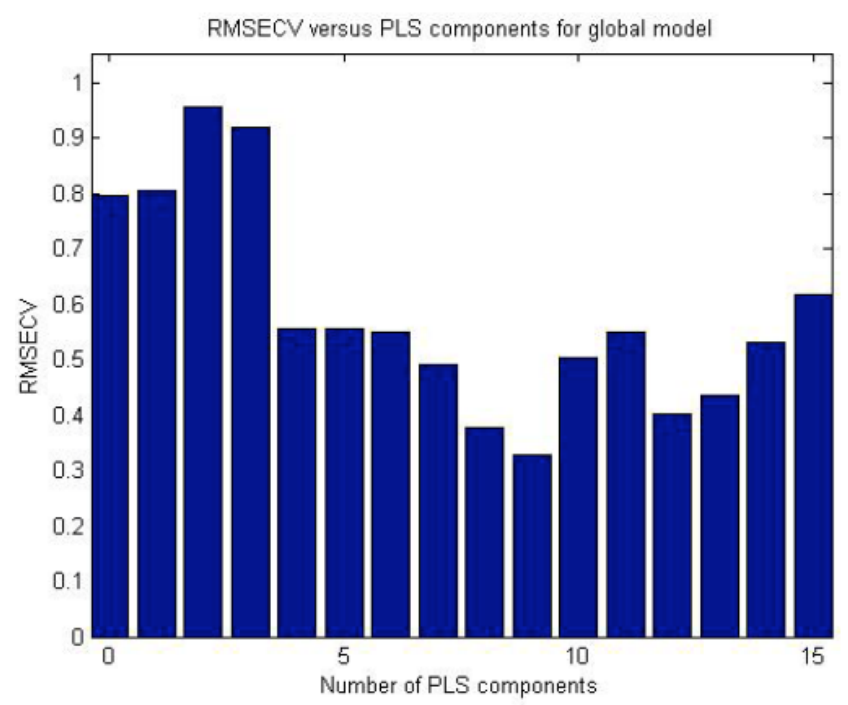

Figure 5: RMSECV versus PLS componentsfor modeling based on the vinegar NIR spectra for acid predication in calibration.

parameters was chosen and for each configuration, the ACO-PLS was run five times for selection of the best subset of wavelengths for determination of TAC. It should be emphasised that the experimental design was employed just for obtaining the most likely configuration in finding the global optimum, and not for fitting of the responses according to an assumed model. The criteria for optimization included the average and standard deviation of PRESS of $50 \%$ of the best ants in each ACO-CLS run. The results obtained from the factorial design process for TAC resulted in the global solution, where $\alpha, \beta$ and $\rho$ values are $0.8,0.8$ and 0.25 , respectively. In Figure $6 \mathrm{a}$ is shown the variation of the RMSECV of the best ant, as obtained by ACO-PLS, as a function of number of iterations. As is obvious, the graph emphasises the fast evolution of the ACO to find the best solution for the system. Figure $6 \mathbf{b}$ shows the predication results of the ACO-PLS model based on the three prominent NIR spectra bands identified (5714 5918, 6125 6331, $7548 \sim 7750 \mathrm{~cm}^{-1}$ ) together with the leave-one-out, cross validation. We also evaluated the performance of the ACO-PLS algorithms using a new dataset (18 Zhenjiang vinegar and 12 Shanxi vinegar) as an independent prediction dataset as shown in Figure 6c. The value of coefficient for prediction $\left(R_{p}\right)$ was 0.921 and the value of root mean square error for prediction (RMSEP) was 0.3031 in predication set.

\section{CONCLUSIONS}

It can be concluded that the NIR spectroscopy technique based on ACO-PLS has high potential to 


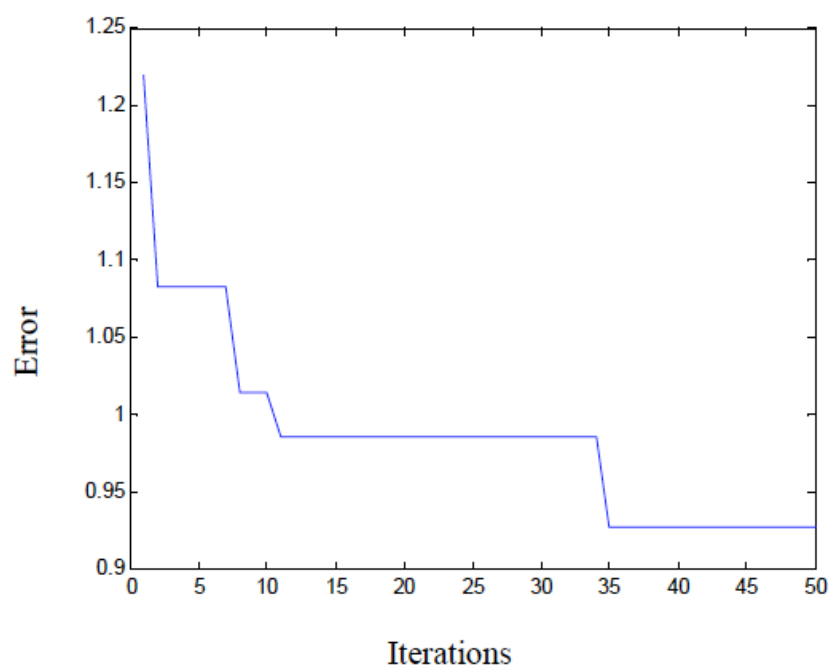

(a)

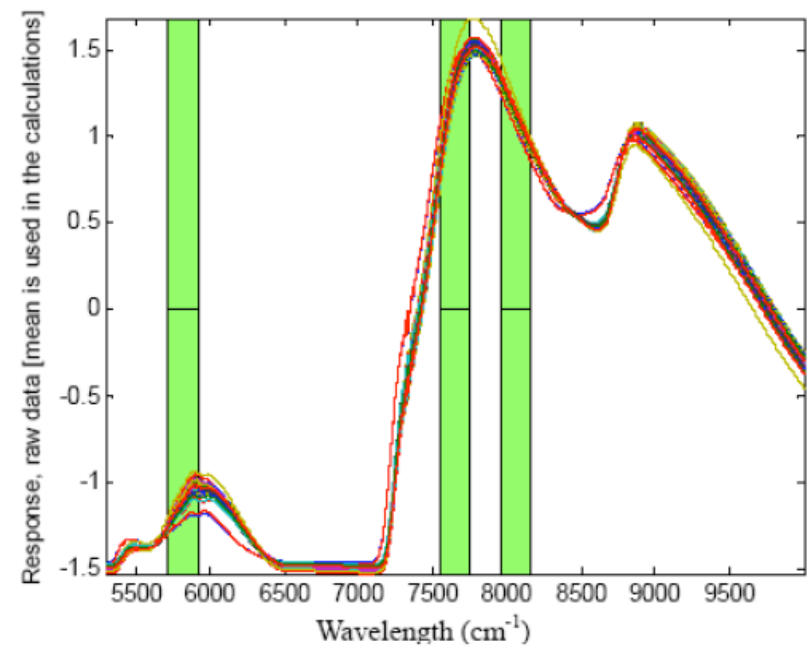

(b)

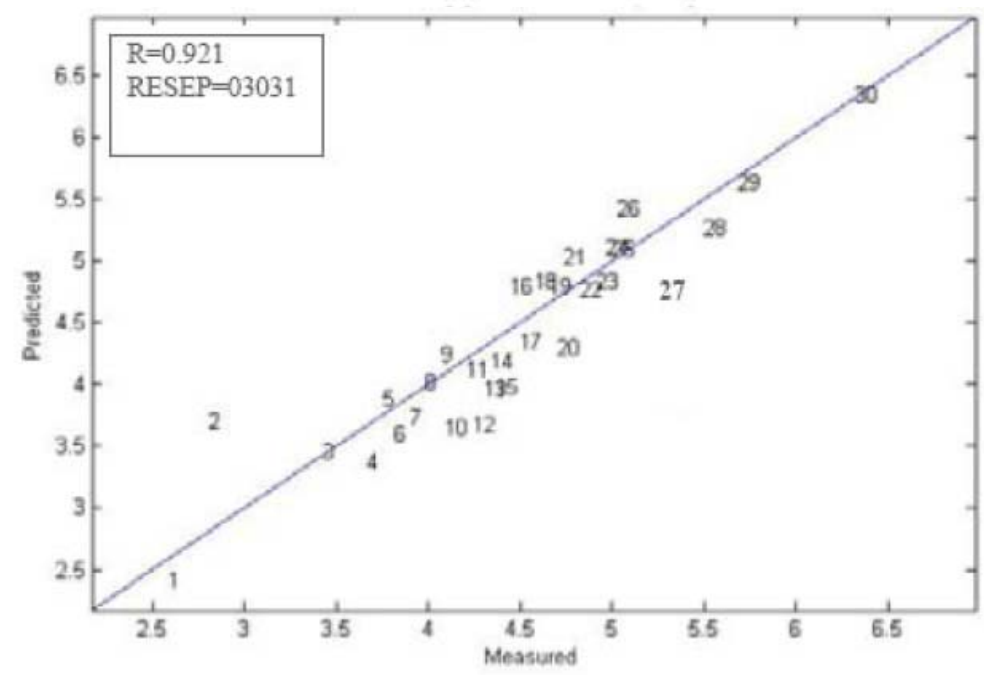

(c)

Figure 6: ACO-PLS modeling based on the vinegar NIR spectra for acid predication. (a) The mean classification error rates of the best performing ants for 50 iterations in ACO-PLS algorithms Parameters (100 ants, $\alpha=0.8, \beta=0.8$ and $\rho=0.25)$, (b) The most significant NIR spectra regions identified by the ACO-PLS algorithms for TAC predication, (c) Scatter plot of measured value and predicated value for prediction set.

identify the vinegar category and to predication total acid content in a nondestructive way. Baonin and Micu vinegar could be distinguished from the other categories vinegar in the two-dimension principal component space. The Zhengjiang vinegar and Shanxi vinegar, which were mixed together, were classified by ACO-PLS. The best results were obtained using two prominent NIR spectra bands identified $\left(5714 \sim 6125 \mathrm{~cm}^{-}\right.$ $\left.1,8366 \sim 8568 \mathrm{~cm}^{-1}\right)$ with ACO-PLS algorithms (100 ants, $\alpha=1, \beta=1$ and $\rho=0.2$ ). They are up to $95.6 \%$ in calibration set; $96.7 \%$ in test set. Total acid contents were used as chemical descriptors to identify the quality level of vinegars. For the predication of total acid content, ACO-PLS regression model produced acceptable precision and accuracy in predicting total acid content based on the spectra data at NIR region.
In contrast to PLS approaches, ACO-PLS algorithm shows its excellent performance in predication results. The overall results sufficiently demonstrate that FT-NIR technique coupled with ACO-PLS pattern recognition can be used to check the vinegar quality.

\section{ACKNOWLEDGEMENTS}

Chinese 863 Program, (Grant No. 2011AA00807), the Chinese Excellence Doctor Foundation (Project Nos. 200968), New Century Excellent Talents in University (NCET-11-0986), Foundation for independent innovation of agricultural sciences in Jiangsu province (CX(11)2028), PAPD, Science and technology support program of Zhenjiang (NY2011026). 


\section{REFERENCES}

[1] Ji-Cheng Chen QCQG. Characterization of Chinese vinegars by electronic nose. Food Chem 2010; 122: 1247-52.

[2] Goodarzi M, Freitas MP, Heyden YV. Linear and nonlinear quantitative structure-activity relationship modeling of the HIV-1 reverse transcriptase inhibiting activities of thiocarbamates. Analyt Chim Acta 2011; 705(1-2): 166-73. http://dx.doi.org/10.1016/i.aca.2011.04.046

[3] Moros J, Inon FA, Garrigues S, de la Guardia A. Determination of vinegar acidity by attenuated total reflectance infrared measurements through the use of second-order absorbance-pH matrices and parallel factor analysis. Talanta 2008; 74: 632-41. http://dx.doi.org/10.1016/j.talanta.2007.06.046

[4] Casale M, Abajo MJS, Sáiz JMG, Pizarro C, Forina M. Study of the aging and oxidation processes of vinegar samples from different origins during storage by near-infrared spectroscopy. Analyt Chim Acta 2006; 557: 360-66. http://dx.doi.org/10.1016/j.aca.2005.10.063

[5] Xu QP, Tao WY, Ao ZH. Antioxidant activity of vinegar melanoidins. Food Chem 2007; 102(3): 841-49.

http://dx.doi.org/10.1016/j.foodchem.2006.06.013

[6] García-Parrillaa MC, Camachob ML, Herediaa FJ, Troncoso AM. Separation and identification of phenolic acids in wine vinegars by HPLC. Food Chem 1994; 50(3): 313-15. http://dx.doi.org/10.1016/0308-8146(94)90140-6

[7] Romero EG, Muñoz GS, Alvarez PJM, Ibáñez MDC. Determination of organic acids in grape musts, wines and vinegars by high-performance liquid chromatography. J Chromatogr A 1993; 655(1): 111-17. http://dx.doi.org/10.1016/0021-9673(93)87018-H

[8] De Vero L, Gala E, Gullo M, Solieri L, Landi S, Giudici P. Application of denaturing gradient gel electrophoresis (DGGE) analysis to evaluate acetic acid bacteria in traditional balsamic vinegar. Food Microbiol 2006; 23(8): 809-13. http://dx.doi.org/10.1016/j.fm.2006.01.006

[9] Plessi M, Bertelli D, Miglietta F. Extraction and identification by GC-MS of phenolic acids in traditional balsamic vinegar from Modena. J Food Comp Anal 2006; 19(1): 49-54. http://dx.doi.org/10.1016/j.jfca.2004.10.008

[10] Cocchi M, Durante C, Grandi M, Lambertini P, Manzinib D, Marchetti A. Simultaneous determination of sugars and organic acids in aged vinegars and chemometric data analysis. Talanta 2006; 69(5): 1166-75. http://dx.doi.org/10.1016/j.talanta.2005.12.032

[11] Sáiz-Abajo MJ, González-Sáiz JM, Pizarro C. Prediction of organic acids and other quality parameters of wine vinegar by near-infrared spectroscopy. A feasibility study. Food Chem 2006; 99; 615-21. http://dx.doi.org/10.1016/j.foodchem.2005.08.006

[12] Fu XG, Yan GZ, Chen B, Li HB. Application of wavelet transforms to improve prediction precision of near infrared spectra. J Food Eng 2005; 69: 461-66.

http://dx.doi.org/10.1016/j.jfoodeng.2004.08.039
[13]

García-Parrilla MC, Heredia FJ, Troncoso AM, González AG. Spectrophotometric determination of total procyanidins in wine vinegars. Talanta 1997; 44: 119-23.

http://dx.doi.org/10.1016/S0039-9140(96)02012-7

[14] Liu F, He Y, Wang L. Comparison of calibrations for the determination of soluble solids content and $\mathrm{pH}$ of rice vinegars using visible and short-wave near infrared spectroscopy. Analyt Chim Acta 2008a; 610: 196-204. http://dx.doi.org/10.1016/..aca.2008.01.039

[15] Liu F, He Y, Wang L. Determination of effective wavelengths for discrimination of fruit vinegars using near infrared spectroscopy and multivariate analysis. Analyt Chim Acta 2008b; 615: 10-17. http://dx.doi.org/10.1016/.aca.2008.03.030

[16] Xiaobo Z, Jiewen Z, Povey MJW, Holmes M, Hanpin M. Variables selection methods in near-infrared spectroscopy. Analyt Chim Acta 2010; 667(1-2): 14-32. http://dx.doi.org/10.1016/i.aca.2010.03.048

[17] Xiaobo Z, Jiewen Z, Xingyi H, Yanxiao L. Use of FT-NIR spectrometry in non-invasive measurements of soluble solid contents (SSC) of 'Fuji' apple based on different PLS models. Chemometr Intellig Lab Syst 2007; 87(1): 43-51. http://dx.doi.org/10.1016/j.chemolab.2006.09.003

[18] Shamsipur M, Zare-Shahabadi V, Hemmateenejad B, Akhond M. Ant colony optimisation: a powerful tool for wavelength selection. J Chemometr 2006; 20(3-4): 146-57. http://dx.doi.org/10.1002/cem.1002

[19] Allegrini F, Olivieri AC. A new and efficient variable selection algorithm based on ant colony optimization. Applications to near infrared spectroscopy/partial least-squares analysis. Analyt Chim Acta 2011; 699(1): 18-25. http://dx.doi.org/10.1016/j.aca.2011.04.061

[20] Pan Y, Jiang JC, Wang R, Jiang JJ. Predicting the net heat of combustion of organic compounds from molecular structures based on ant colony optimization. J Loss Preven Proc Ind 2011; 24(1): 85-89. http://dx.doi.org/10.1016/j.jp.2010.11.001

[21] Goodarzi M, Freitas MP, Jensen R. Ant colony optimization as a feature selection method in the QSAR modeling of antiHIV-1 activities of 3-(3,5-dimethylbenzyl)uracil derivatives using MLR, PLS and SVM regressions. Chemometr Intellig Lab Syst 2009; 98(2): 123-29. http://dx.doi.org/10.1016/i.chemolab.2009.05.005

[22] Tang $\mathrm{Y}, \mathrm{Wu} \mathrm{M}$. The simultaneous separation and determination of five organic acids in food by capillary electrophoresis. Food Chem 2005; 103(1): 243-48. http://dx.doi.org/10.1016/j.foodchem.2005.09.022

[23] Workman J, Weyer L. Practical guide to interpretive nearinfrared spectroscopy. Boca Raton: CRC Press 2007. 